\title{
Angiostrongylus (Parastrongylus) cantonensis en huéspedes intermediarios y definitivos en Ecuador, 2014-2017
}

\author{
Luis Solórzano-Alava ${ }^{1}$, Francisco Sánchez-Amador ${ }^{1}$, Talía Valverde ${ }^{2}$ \\ ${ }^{1}$ Centro de Referencia Nacional de Parasitología, Instituto Nacional de Investigación en Salud \\ Pública "Leopoldo Izquieta Pérez", Guayaquil, Ecuador \\ ${ }^{2}$ Centro de Especialidades Letamendi, Instituto Ecuatoriano de Seguridad Social, Guayaquil, \\ Ecuador
}

Introducción. Angiostrongylus cantonensis es un serio problema de salud pública y está ampliamente distribuido en el país. Cuando el parásito infecta a los caracoles terrestres Achatina, se enquista en su interior y puede infectar accidentalmente a las personas y otros mamíferos.

Objetivo. Establecer la distribución geográfica de $A$. cantonensis en huéspedes intermediarios (Achatina fulica) y definitivos (Rattus spp.) en Ecuador entre el 2014 y el 2017.

Materiales y métodos. Se recolectaron 2.908 ejemplares de $A$. fulica en 16 provincias utilizando el método de captura por unidad de esfuerzo durante 30 minutos. Se capturaron 211 ejemplares de las especies huéspedes, de los cuales 20 eran Rattus rattus y $191 R$. norvegicus. Los ejemplares fueron transportados para su análisis al Instituto Nacional de Salud Pública e Investigación en Guayaquil.

En los tejidos del caracol se identificaron y se contabilizaron larvas L3 que luego se inocularon en ratas de laboratorio para reproducir el ciclo de vida. En los roedores se disecaron los cerebros, los corazones, las arterias pulmonares y los pulmones, y se identificaron los parásitos por morfología taxonómica.

Resultados. De los caracoles recolectados se encontraron 441 positivos para $A$. cantonensis (15,2\%) y un total de 6.166 larvas L3. En los ejemplaresRattus spp. capturados (211), 77 (36,5\%) estaban infectados con $A$. cantonensis, con un total de 220 parásitos (larvas L4-L5 y adultos).

Conclusiones. Se constató la presencia de A. cantonensis en varias provincias, lo que confirma el carácter endémico de esta zoonosis en el territorio nacional. La presencia de Rattus spp., huésped definitivo del parásito, y de huéspedes intermediarios, indica el potencial zoonótico de esta infección parasitaria.

Recibido: $16 / 05 / 18$

Publicado: $11 / 10 / 18$

\section{Citación:}

Solórzano-Alava L, Sánchez-Amador F, Valverde T. Angiostrongylus (Parastrongylus) cantonensis en huéspedes intermediarios y definitivos en Ecuador, 2014-2017. Biomédica. 2019;39:370-84. https://doi.org/10.7705/biomedica.v39i3.4387

\section{Correspondencia:}

Luis Fernando Solórzano, Centro de Referencia Nacional de Parasitología, Instituto Nacional de Investigación en Salud Pública "Leopoldo Izquieta Pérez", Avenida Julián Coronel 905 Esmeraldas y José Mascote, Guayaquil, Ecuador Teléfono: (+593) 42288096

luisfernando.solorzano@gmail.com

\section{Contribución de los autores:}

Luis Fernando Solórzano: obtención del parásito

$A$. cantonensis en caracoles y ratas

Todos los autores participaron en el diseño metodológico, la recolección y la captura de moluscos y roedores, el análisis de datos y la escritura del manuscrito.

Financiación:

El presente estudio fue financiado por los propios autores.

Conflicto de intereses:

No se declaró ninguno.
Palabras clave: Angiostrongylus cantonensis; ratas; interacciones huésped-parásitos; Ecuador.

\section{Angiostrongylus (Parastrongylus) cantonensis on intermediate and definitive hosts} in Ecuador, 2014-2017

Introduction: Angiostrongylus cantonensis is a serious public health problem and is widely distributed in the country. When the parasite infects the snails, it becomes deeply embedded in their interior and accidentally, it can infect people and other mammals. Objective: To establish the geographical distribution of $A$. cantonensis intermediate hosts (Achatina fulica) and definitive hosts (Rattus spp.) in Ecuador from 2014 to 2017. Materials and methods: We collected 2,908 A. fulica specimens in 16 provinces using the capture method per unit of effort for 30 minutes. We captured 211 hosts of which 20 were Rattus rattus and 191 R. norvegicus. The specimens were transported to the Instituto Nacional de Salud Pública e Investigación in Guayaquil where the larvae L3 were identified and counted in the tissues of the snail, which were then inoculated in laboratory rats to reproduce the life cycle. In the rodents, the brains, hearts, lung arteries and lungs were dissected, and the parasites were identified by taxonomic morphology.

Results: Of the snails harvested, 441 were positive for $A$. cantonensis (15.2\%) and a total of 6,166 L3 larvae were found; 77 (36.5\%) specimens of Rattus spp., were infected with $A$. cantonensis and a total of 220 parasites (L4-L5 larvae and adult worms) were collected. Conclusions: We confirmed the presence of $A$. cantonensis in several provinces, which ratifies the endemic nature of this zoonosis in the national territory. Rattus spp. specimens constitute the definitive hosts of the parasite, which together with the presence of intermediate hosts, indicates the zoonotic potential of this parasitic infection.

Keywords: Angiostrongylus cantonensis; rats; host-parasite interactions; Ecuador. 
Angiostrongylus cantonensis, un parásito zoonótico transmitido en los alimentos, se ha reconocido como el agente patógeno primario asociado con la meningitis eosinofílica humana o meningoencefalitis eosinofílica. Se han informado miles de casos de meningitis eosinofílica causada por este parásito en todo el mundo. La angiostrongiliasis es una enfermedad de importancia creciente en salud pública, ya que la globalización contribuye a su diseminación y a la infección de huéspedes vulnerables (1).

El parásito está ampliamente distribuido en Ecuador y constituye un serio problema de salud pública. Cuando $A$. cantonensis infecta a los caracoles, el parásito se aloja en la cavidad paleal de su manto. Las personas y mamíferos superiores se infectan directa e indirectamente al manipular o ingerir caracoles crudos o por alimentos contaminados por ellos (2).

Angiostrongylus cantonensis fue descrito por primera vez en 1935. Se encontró en los pulmones de Rattus rattus y $R$. norvegicus capturados en Cantón, China, por lo que se le reportó como Pulmonema cantonensis (3). En 1981 se demostró un caso clínico de meningitis eosinofílica causado por $A$. cantonensis en Cuba (4) y en la década de los sesenta la enfermedad apareció en Okinawa, en las islas Ryukyu, Honshu y Kyushu, en Nueva Bretaña, Samoa Americana y Samoa Occidental, Australia, Fiji y en China continental (5).

La infección se ha presentado principalmente en la cuenca del Pacífico, en Hawai (6), y en el sudeste asiático, en Tailandia, Vanuatu, Islas Marshall, Islas Carolinas, Islas Cook, Filipinas, Nueva Caledonia y Tahití (7).

En Cuba, se llevaron a cabo diversos estudios en la década de los años 80. En la provincia de Camagüey se demostró que más del $60 \%$ de los moluscos recolectados vivos, pertenecientes a cuatro especies, estaban infectados de forma natural con larvas L3 de A. cantonensis (8).

En Brasil se han encontrado moluscos terrestres con infección natural por A. cantonensis en las regiones del nordeste y del sureste $(9,10)$.

En el 2008 se describió el primer foco de transmisión natural de $A$. cantonensis en Ecuador y actualmente el parásito es endémico en este país. Uno de sus principales huéspedes intermediarios es el también llamado caracol gigante africano (Achatina fulica). Este molusco habita en las zonas urbanas y en las rurales, y es uno de los principales transmisores de meningoencefalitis eosinofílica y angiostrongiliasis ocular por alimentos contaminados o por la ingestión del caracol crudo (11).

En ese mismo año se reportaron diferentes brotes de angiostrongilosis en los recintos La Ercilia y Sabanetilla en el cantón Ventanas de la provincia de Los Ríos, y en la provincia de Santo Domingo de los Tsáchilas, y se encontró el parásito en caracoles terrestres ( $A$. fulica) y acuáticos (Pomacea spp.), así como en roedores (Rattus spp.) (11,12).

La infección por $A$. cantonensis es la causa más común de meningitis eosinofílica infecciosa en humanos y produce angiostrongiliasis en el sistema nervioso central. En el Caribe, el parásito se ha encontrado en en las Antillas Mayores, en Cuba, República Dominicana, Haití, Puerto Rico y Jamaica, y en las Antillas Menores, en Granada y Guadalupe (13).

El objetivo de este trabajo fue establecer la distribución geográfica del parásito en huéspedes intermediarios (caracoles) y definitivos (roedores) en Ecuador entre el 2014 y el 2017. El conocimiento de la distribución geográfica 
de los huéspedes intermediarios y definitivos contribuirá al establecimiento de medidas puntuales en esas zonas para prevenir la aparición de nuevos casos.

\section{Materiales y métodos}

Entre enero del 2014 y diciembre del 2017 se recolectaron manualmente 2.908 ejemplares de caracol gigante africano $(A$. fulica) con el método de captura por unidad de esfuerzo durante 30 minutos $(14,15)$. Posteriormente, fueron puestos en recipientes plásticos, etiquetados (origen, cantidad y especies) y transportados al Instituto Nacional en Salud Pública e Investigación "Leopoldo Izquieta Pérez" en Guayaquil (15).

La recolección se llevó a cabo en 16 provincias continentales, a saber: Guayas, Santa Elena, Santo Domingo de los Tsáchilas, Napo, Los Ríos, Chimborazo, Pichincha, El Oro, Cañar, Sucumbíos, Francisco de Orellana, Pastaza, Zamora Chinchipe, Esmeraldas, Bolívar y Manabí.

Los caracoles se examinaron en busca de larvas L3 de $A$. cantonensis empleando el método modificado de Lobato-Paraense para la extracción de los órganos blandos de los moluscos (15). Estos se sumergieron en una solución de digestión (16) y se observaron para identificar y contabilizar las larvas (L3) según las características taxonómicas de Cowie y Thiengo, et al. $(17,18)$, usando un estereomicroscopio Motic SMZ-168 ${ }^{\text {TM }}$. A continuación, fueron inoculadas en ratas albinas adultas hembras ( $R$. norvegicus) para hacer el seguimiento del ciclo biológico en el Centro de Referencia Nacional de Parasitología (8) (figura 1).

En la captura de las ratas silvestres se usaron nueve trampas Tomahawk $(15 \times 15 \times 45 \mathrm{~cm})$, colocadas estratégicamente durante la noche en lugares donde había indicios de la presencia de roedores, tales como heces, materiales roídos y rincones oscuros, las cuales se revisaban temprano en la mañana siguiente (19).

Posteriormente, se examinaron las heces para observar si había larvas L1 de $A$. cantonensis. Se examinó la cavidad torácica (corazón, arterias pulmonares y pulmones) en busca de los nematodos parásitos, juveniles 0 adultos. Se contaron los nematodos adultos encontrados en la luz de la arteria pulmonar y se guardaron en una solución de formaldehído al 3 \% (11) (figuras 2, 3 y 4).

La morfología de la bursa y el tamaño de las espículas caudales se utilizaron como características taxonómicas para la identificación (4). Se practicó la craneotomía de la bóveda cefálica, de la cual se extrajo el encéfalo para la búsqueda de ninfas L4-L5 de A. cantonensis. Se estableció la prevalencia de ratas con el parásito $A$. cantonensis tanto en estadio larvario L4-L5 como en los estadios adultos (machos y hembras), y el número total de ratas capturadas por provincia $(8,10)$.

\section{Consideraciones éticas}

Este estudio obtuvo los permisos legales contemplados en la Plataforma del Bioterio del Instituto Nacional de Salud Pública e Investigación en Guayaquil (Ecuador) y se ajustó a las normas de la "Guía de manejo y cuidado de animales de laboratorio” del Ministerio de Salud de Perú, 2008 (20). 


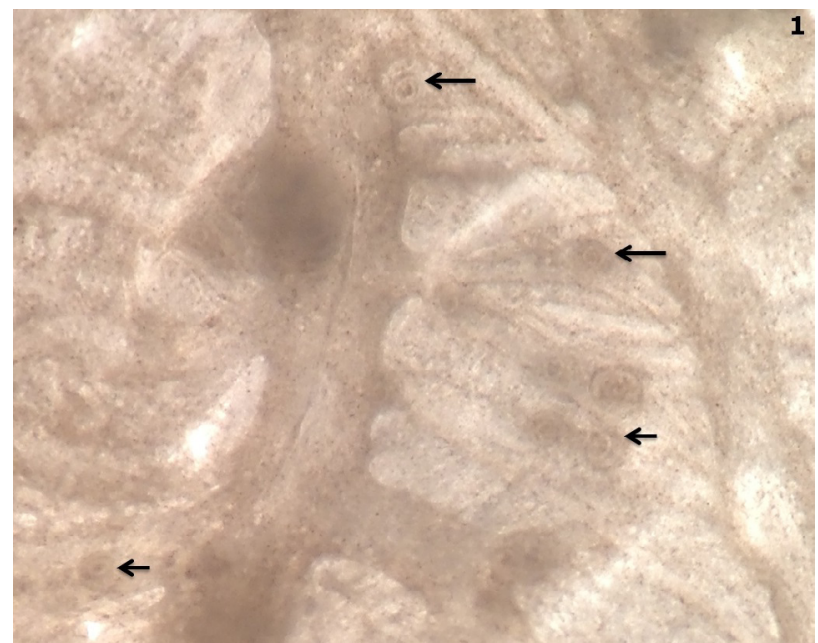

Figura 1. Larvas L2 y L3 de Angiostrongylus cantonensis, enquistadas el interior de la membrana paleal de Achatina fulica.

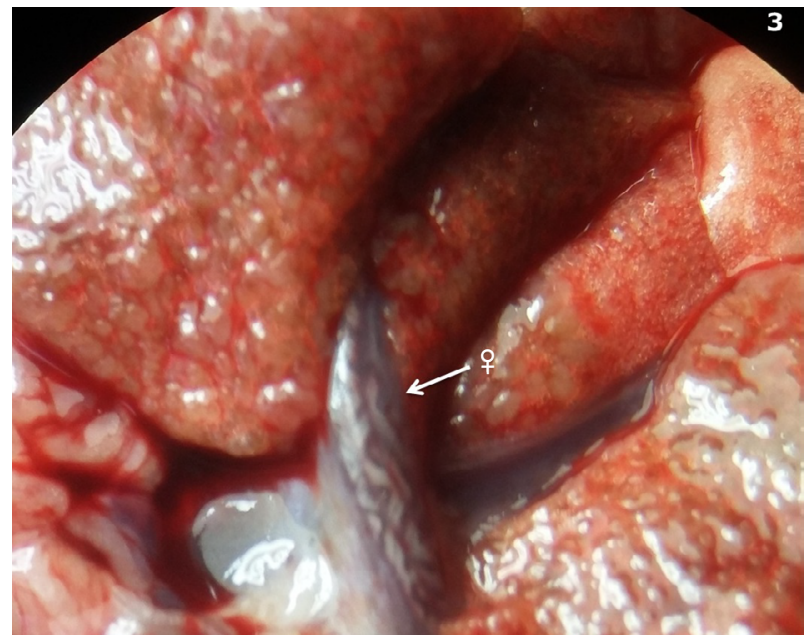

Figura 3. Hembras adultas de Angiostrongylus cantonensis emergiendo a partir de las arterias pulmonares de Rattus norvegicus

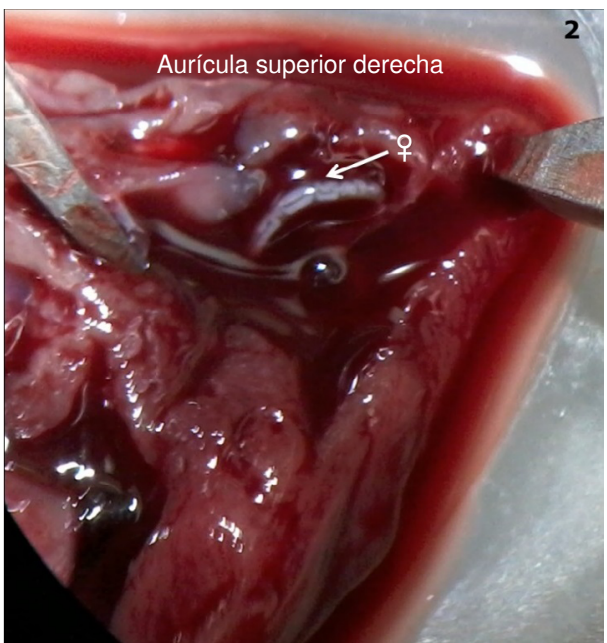

Figura 2. Hembra adulta Angiostrongylus cantonensis en el interior de la aurícula superior derecha de Rattus norvegicus

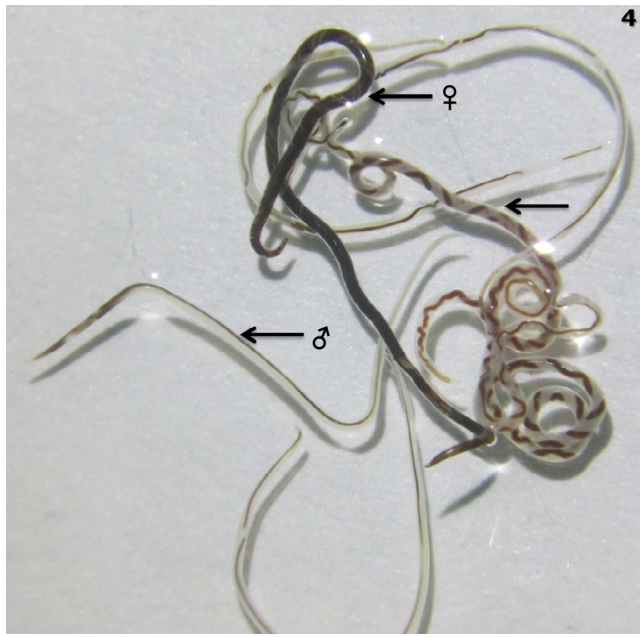

Figura 4. Adultos machos y hembras de Angiostrongylus cantonensis de Rattus norvegicus. El gusano más oscuro corresponde a una hembra de mayor edad.

\section{Resultados}

Se encontraron 441 caracoles positivos para $A$. cantonensis, con una prevalencia total de $15,2 \%$ durante los tres años en las 16 provincias continentales seleccionadas. Solamente en una provincia (Manabí) no se encontró $A$. cantonensis en el interior de los moluscos (cuadro 1).

En el 2017, se encontraron 6.166 larvas L3 de A. cantonensis en el interior de $A$. fulica. En el cuadro 2 y en la figura 5 se presenta la información de la recolección por cantón perteneciente a cada provincia y según el número de caracoles recolectados, el número de caracoles parasitados por $A$. cantonensis y el número total de larvas que se encontraron en su interior.

Los valores por provincia se establecieron en tres categorías por rangos del número de larvas en el interior del manto del caracol: I, de 0-19; II, de 2071, y III, 72-150 (cuadro 3). 
Cuadro 1. Caracoles gigantes africanos (Achatina fulica), caracoles con Angiostrongylus cantonensis presentes y número de parásitos por provincia en Ecuador

\begin{tabular}{lcccc}
\hline Provincias & $\begin{array}{c}\text { Caracoles } \\
(\mathbf{n})\end{array}$ & $\begin{array}{c}\text { Caracoles positivos } \\
(\mathbf{n})\end{array}$ & $\begin{array}{c}\text { Larvas L3 } \\
(\mathbf{n})\end{array}$ & $\begin{array}{c}\text { Prevalencia * } \\
\text { (\%) }\end{array}$ \\
\hline Guayas & 263 & 24 & 1617 & 9,1 \\
Santa Elena & 50 & 4 & 17 & 8,0 \\
Napo & 324 & 88 & 228 & 27,2 \\
Santo Domingo de los Tsáchilas & 234 & 80 & 292 & 34,2 \\
Los Ríos & 449 & 28 & 3.092 & 6,2 \\
Chimborazo & 112 & 31 & 27 & 27,7 \\
Pichincha & 20 & 2 & 14 & 10,0 \\
El Oro & 168 & 49 & 44 & 29,2 \\
Sucumbíos & 132 & 32 & 128 & 24,2 \\
Francisco de Orellana & 197 & 34 & 193 & 17,3 \\
Pastaza & 108 & 9 & 35 & 8,3 \\
Cañar & 320 & 44 & 319 & 13,8 \\
Esmeraldas & 210 & 11 & 69 & 5,2 \\
Bolívar & 41 & 2 & 70 & 4,9 \\
Zamora Chinchipe & 35 & 3 & 21 & 8,6 \\
Manabí & 245 & 0 & 0 & 0,0 \\
Total & 2.908 & 441 & 6.166 & 15,2 \\
\hline
\end{tabular}

* Prevalencia de positividad de Achatina fulica

Cuadro 2. Número de caracoles Achatina fulica, caracoles positivos para Angiostrongylus cantonensis y cantidad de parásitos encontrados en cada cantón

\begin{tabular}{|c|c|c|c|c|}
\hline No. & Cantones - Recintos & $\begin{array}{c}\text { Caracoles } \\
\text { (n) }\end{array}$ & $\begin{array}{c}\text { Positivos } \\
\text { (n) }\end{array}$ & $\begin{array}{c}\text { Parásitos } \\
\text { (n) }\end{array}$ \\
\hline & Costa - Región Litoral & & & \\
\hline 1 & Guayaquil - Guayas & 106 & 0 & 0 \\
\hline 2 & AB Moreno - Guayas & 129 & 24 & 1.617 \\
\hline 3 & Daule - Guayas & 28 & 0 & 0 \\
\hline 4 & Olón - Santa Elena & 25 & 2 & 15 \\
\hline 5 & Manglar Alto - Santa Elena & 25 & 2 & 2 \\
\hline 6 & Esmeraldas - Esmeraldas & 210 & 11 & 69 \\
\hline 7 & El Guabo - El Oro & 144 & 46 & 44 \\
\hline 8 & Pasaje - El Oro & 24 & 3 & 7 \\
\hline 9 & Chone - Manabí & 245 & 0 & 0 \\
\hline 10 & Babahoyo - Los Ríos & 244 & 5 & 5 \\
\hline 11 & Montalvo - Los Ríos & 150 & 21 & 3.085 \\
\hline \multirow[t]{2}{*}{12} & Mata de Cacao - Los Ríos & 55 & 2 & 2 \\
\hline & Sierra - Región Interandina & & & \\
\hline 13 & La Troncal - Cañar & 320 & 44 & 319 \\
\hline 14 & Santo Domingo de los Tsáchilas & 234 & 80 & 292 \\
\hline 15 & Cascajal - Chimborazo & 112 & 31 & 27 \\
\hline 16 & S Miguel de Los Bancos - Pichincha & 20 & 2 & 14 \\
\hline \multirow[t]{2}{*}{17} & Echeandía - Bolívar & 41 & 2 & 70 \\
\hline & Amazonia - Región Oriental & & & \\
\hline 18 & Lago Agrio - Sucumbíos & 42 & 14 & 80 \\
\hline 19 & Sushufindi - Sucumbíos & 64 & 14 & 41 \\
\hline 20 & Joya de los Sachas - Orellana & 94 & 18 & 117 \\
\hline 21 & Fco. de Orellana - Orellana & 61 & 12 & 55 \\
\hline 22 & Loreto - Orellana & 68 & 8 & 28 \\
\hline 23 & Archidona - Napo & 64 & 11 & 150 \\
\hline 24 & Tena - Napo & 60 & 6 & 6 \\
\hline 25 & Carlos Arosemena Tola - Napo & 200 & 71 & 72 \\
\hline 26 & Santa Clara - Pastaza & 56 & 6 & 26 \\
\hline 27 & Puyo - Pastaza & 52 & 3 & 9 \\
\hline 28 & Zamora Chinchipe - Zamora & 35 & 3 & 17 \\
\hline
\end{tabular}




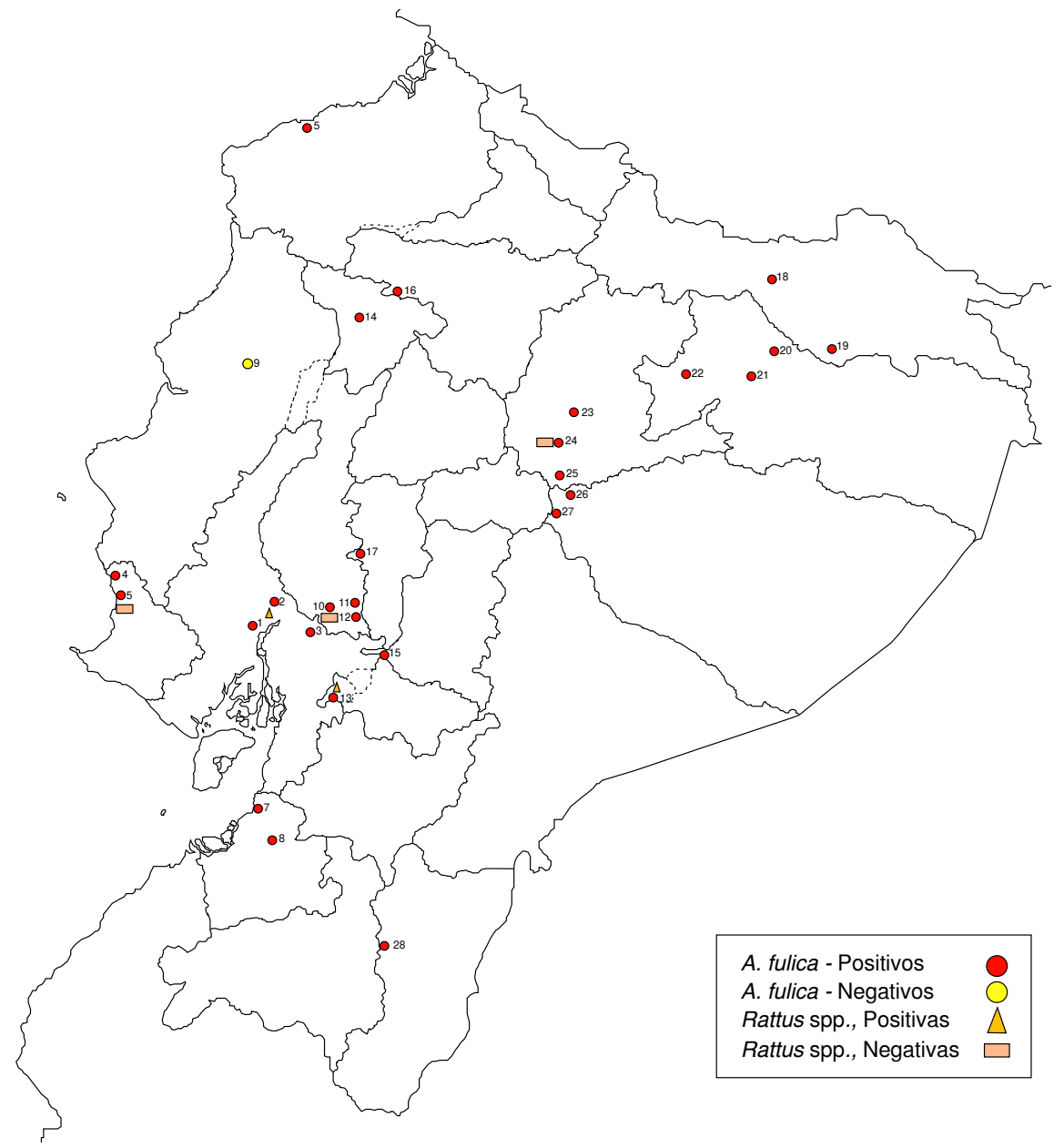

(1) Guayaquil; (2) AB Moreno; (3) Daule; (4) Olón; (5) Manglar Alto; (6) Esmeraldas; (7) El Guabo; (8) Pasaje; (9) Chone; (10) Babahoyo; (11) Montalvo; (12) Mata de Cacao; (13) La Troncal; (14) Santo Domingo; (15) Cascajal; (16) SM de los Bancos; (17) Echeandía; (18) Lago Agrio; (19) Sushufindi; (20) Joya de los Sachas; (21) Fco. Orellana; (22) Loreto; (23) Archidona; (24) Tena; (25) Carlos Arosemena Tola; (26) Santa Clara; (27) Puyo; (28) Zamora

Figuras 5. Mapa político del Ecuador con los puntos de referencia donde se capturaron caracoles (Achatina fulica) y roedores (Rattus spp.) positivos para Angiostrongylus cantonensis y negativos para el parásito

Cuadro 3. Cantidad de parásitos Angiostrongylus cantonensis en caracoles gigantes africanos (Achatina fulica) en diferentes provincias de Ecuador, divididas en tres categorías según el número de larvas en el interior del manto del caracol: I, de 0-19; II, de 20-71, y III, 72-150 (2017)

\begin{tabular}{lccc}
\hline Provincias & Categoría I & Categoría II & Categoría III \\
\hline Guayas & 6 & 10 & 8 \\
Santa Elena & 2 & 2 & 0 \\
Napo & 82 & 6 & 0 \\
Santo Domingo de los Tsáchilas & 37 & 2 & 1 \\
Los Ríos & 12 & 4 & 12 \\
Chimborazo & 16 & 6 & 0 \\
Pichincha & 2 & 0 & 0 \\
El Oro & 39 & 5 & 0 \\
Sucumbíos & 30 & 2 & 0 \\
Orellana & 33 & 1 & 0 \\
Pastaza & 9 & 0 & 0 \\
Cañar & 22 & 2 & 1 \\
Esmeraldas & 8 & 1 & 0 \\
Manabí & 0 & 0 & 0 \\
\hline
\end{tabular}


Se identificaron 20 especímenes de $R$. rattus y 191 de $R$. norvegicus, que se analizaron en Guayaquil en el Centro de Referencia Nacional de Parasitología del Instituto Nacional de Salud Pública e Investigación.

De los 20 ejemplares de $R$. rattus, siete se encontraron infectados con $A$. cantonensis, con una prevalencia de $35 \%$ y 30 parásitos recolectados. De los 191 especímenes de $R$. norvegicus, 68 se encontraron infectados con $A$. cantonensis, para una prevalencia de $35,6 \%$ en un total de 220 organismos recolectados. De los 211 especímenes, en uno de $R$. norvegicus se encontró el parásito en la bóveda cefálica en estadio larvario L4-L5, con un total de 62 organismos; en el corazón de un ejemplar de $R$. norvegicus se encontró un ejemplar en estadio L5 y adulto (cuadro 4 y figura 5).

Los parásitos se encontraron en la aurícula derecha del corazón en la mayoría (55/68) de los casos. En el corazón y en los pulmones se observaron algunas zonas hemorrágicas, fibrosis y abscesos.

En la Provincia del Guayas se encontraron ratas positivas para $A$. cantonensis, de las cuales siete eran $R$. rattus y $55 R$. norvegicus. En las provincias de Los Ríos y Cañar se encontraron un solo roedor positivo y uno negativo, respectivamente. No se encontraron roedores positivos en la provincia de Santa Elena, ni en Napo (cuadro 4).

Cuadro 4. Prevalencia de Angiostrongylus cantonensis en Rattus spp., por provincias, total de parásitos, roedores negativos, total general y porcentajes

\begin{tabular}{llccccc}
\hline No. & Provincias - Cantón & $\begin{array}{c}\text { Angiostrongylus } \\
\text { cantonensis } \\
(\mathbf{n})\end{array}$ & $\begin{array}{c}\text { Parásitos } \\
\mathbf{( n )}\end{array}$ & $\begin{array}{c}\text { Negativas } \\
(\mathbf{n})\end{array}$ & $\begin{array}{c}\text { Total } \\
(\mathbf{n})\end{array}$ & $\begin{array}{c}\text { Porcentaje } \\
(\%)\end{array}$ \\
\hline 1 & Guayas - Guayaquil & 75 & 213 & 125 & 200 & 37,5 \\
10 & Los Ríos - Babahoyo & 1 & 2 & 1 & 2 & 50,0 \\
13 & Cañar/La Troncal & 1 & 5 & 1 & 2 & 50,0 \\
4 & Santa Elena/Olón & 0 & 0 & 5 & 5 & 0,0 \\
25 & Napo/Tena & 0 & 0 & 2 & 2 & 0,0 \\
& Total & 77 & 220 & 134 & 211 & 36,5 \\
\hline
\end{tabular}

\section{Discusión}

En estudios hechos en moluscos en 18 condados de Florida, Estados Unidos, entre el 2013 y el 2015, se hallaron 1.437 huéspedes intermediarios (caracoles) pertenecientes a 32 especies de gasterópodos, de los cuales $27(1,9 \%)$ fueron positivos para $A$. cantonensis. Las muestras fueron confirmadas por reacción en cadena de la polimerasa (PCR) (21).

En los trabajos en $A$. fulica llevados a cabo en nueve municipios que forman la Baixada Santista de São Paulo, Brasil, se encontró que de 540 caracoles analizados, 117 (21,7\%) estaban infectados con $A$. cantonensis (22).

Los estudios efectuados en los moluscos $T$. pisana y $P$. lamarckii en el 2015 en Tenerife, Islas Canarias, España, reportaron 19,3\% de larvas L3 de $A$. cantonensis, confirmados por el método de Loop-mediated Isothermal Amplification, LAMP (23).

En un estudio realizado entre el 2010 y el 2011 en el subdistrito de ThaPho, Phitsanulok, Tailandia, 38 de 307 caracoles $A$. fulica presentaron $A$. cantonensis, es decir, una tasa de infección general de 12,38 \% (24). 
Los estudios realizados en Ecuador durante el periodo de 2008 a 2011 en seis de las 10 provincias previamente seleccionadas para este estudio, se encontraron ejemplares de $A$. fulica infectados con $A$. cantonensis $(2,11,12)$. Posteriormente, durante el 2012 , en diez de las catorce provincias seleccionadas se detectó la presencia de $A$. cantonensis en los caracoles. De un total de 2.784 caracoles, 1.054 correspondieron al género Pomacea spp., y 1.730 a Achatina sp., y la positividad total para el parásito fue de 11,2 \% (11).

En el presente estudio de tres años, se encontró un 15,2\% de caracoles infectados con $A$. cantonensis, lo que evidencia un incremento en comparación con los estudios anteriores. Se constató su presencia en las mismas provincias estudiadas previamente (2008-2012) y en ocho más: Santa Elena, Napo, Pichincha, Sucumbíos, Francisco de Orellana, Pastaza, Cañar y Zamora Chinchipe.

En Ecuador este caracol exótico se introdujo en la provincia de Esmeraldas en el $2005(8,11,12,15)$, probablemente traído de Asia con fines comerciales que no tuvieron éxito. Su gran capacidad reproductiva y la tendencia de las personas a liberarlos en la naturaleza son, probablemente, las razones de la rápida invasión de esta especie (15) que hoy se encuentra en la mayoría de las provincias de Ecuador. Sus voraces hábitos alimenticios, entre otros factores, contribuyen al exterminio de la flora y la fauna endémicas, reducen los recursos disponibles y aumentan la competencia por el espacio físico.

El aumento de la presencia de $A$. cantonensis en el país probablemente es el resultado de la rápida propagación de su huésped intermediario, $A$. fulica, lo que contribuye a la dispersión de este parásito y a la infección del huésped definitivo.

En el estudio en ratas silvestres (Rattus spp.) en tres de los 18 condados seleccionados en Florida, Estados Unidos, entre el 2013 y el 2015, se encontró que de 171 ejemplares de $R$. rattus recolectados, 39 (22,8\%) estaban infectados con $A$. cantonensis (21).

En los estudios en Tenerife, España, se encontró una seroprevalencia en ratas de 55,6\% determinada mediante el método de ELISA (23 ).

La prevalencia de $A$. cantonensis en ejemplares de $R$. norvegicus en Río de Janeiro fue relativamente alta en 146 ratas, es decir, una prevalencia de $71 \%$, mayor que en otros estudios anteriores en Brasil (25).

En áreas de Cuba y República Dominicana se han registrado porcentajes altos $(60 \% ; 12 / 20)$ de ratas infectadas con $A$. cantonensis (26).

Entre el 2008 y el 2011, en Ecuador se encontró una prevalencia de $11 \%$ en ejemplares de Rattus spp. en dos provincias $(11,12)$. En el presente estudio de tres años, se capturaron ejemplares de Rattus spp. en 5 de las 16 provincias seleccionadas (incluidas las provincias estudiadas entres el 2008 y el 2012), en las cuales se registró una prevalencia de 37,5\%, en las zonas urbanas más habitadas de la provincia de Guayas.

En la isla de Hawái, el 72,7 \% (396/545) de los ejemplares de Rattus spp. analizados estaba infectado con lombrices adultas, y el 93,9\% (512/545) de las ratas resultó positivo para la infección por $A$. cantonensis determinado por la presencia de gusanos adultos vivos, gusanos adultos enquistados, larvas L3 o por PCR en tejido cerebral. 
La prevalencia excepcionalmente alta de la infección por $A$. cantonensis en Rattus spp. en la isla de Hawái es motivo de preocupación e indica que el potencial de infección humana con esta zoonosis emergente es mayor de lo que se pensaba anteriormente (27).

Sin embargo, el reducido tamaño de la muestra en estos estudios a corto plazo no permitió obtener resultados concluyentes e impidió la inferencia de la estructura (25). En el presente estudio se observó una prevalencia alta y estable de $A$. cantonensis en los tres años en comparación con los estudios anteriores $(11,12)$.

Esta prevalencia estable también podría explicarse por los siguientes hechos: una larga vida del parásito en el huésped definitivo y la liberación de larvas L1 durante prolongados períodos, lo cual puede mantener la transmisión del parásito al huésped intermedio incluso en tiempos de baja abundancia del caracol en el entorno $(25,26)$; la presencia de más de una especie de molusco, lo que puede facilitar el desarrollo de la fase larvaria infecciosa L3 $(25,29)$; la ausencia de factores que limiten la abundancia de la rata (por ejemplo, disponibilidad de refugio constante y de alimentos), y la heterogeneidad genética de $A$. cantonensis, que puede facilitar la adaptación a nuevos entornos $(25,28,30)$.

La meningitis eosinofílica se considera una zoonosis parasitaria que afecta a las ratas, el huésped definitivo, en el cual los gusanos adultos hembras y machos se localizan en las arterias pulmonares. Después de la cópula, las hembras ponen sus huevos que luego eclosionan en las ramas terminales de las arterias pulmonares y producen larvas de primer estadio (L1). Estas larvas migran a la tráquea, son deglutidas, pasan al tracto gastrointestinal y son eliminadas en las heces. Al ser ingeridas por moluscos (caracoles terrestres y marinos), que son huéspedes intermediarios, experimentan dos mudas hasta convertirse en larvas del tercer estadio (L3), la forma infecciosa para el hombre y otros mamíferos, los cuales completan el ciclo al ingerir los caracoles infectados. Las larvas penetran la pared intestinal y migran por el sistema circulatorio, y en el transcurso de dos a tres días llegan al cerebro, donde mudan dos veces hasta convertirse en larvas de cuarto (L4) y quinto estadio (L5) (adultos jóvenes). Posteriormente, los parásitos migran a la arteria pulmonar y a las cavidades del corazón derecho, el asentamiento en este último hábitat se produce aproximadamente cuatro semanas después de la ingestión de larvas L3 (8).

Desde la primera notificación de infección humana por $A$. cantonensis en Taiwán, en 1945, se han informado brotes importantes en China continental y se han registrado casos en diferentes regiones del mundo, aproximadamente en 30 países. La amenaza no es solo para las personas que viven en las zonas endémicas, sino también para aquellas que visitan estas regiones. Como ya se mencionó, el parásito es endémico en el sur de Asia, en las islas del Pacífico y del Caribe y en partes de Australia, el sudeste de Estados Unidos, Egipto, Nigeria, Costa de Marfil y Sudamérica (Brasil y Ecuador). La infección por $A$. cantonensis ha atraído cada vez más atención debido a los brotes y a un número creciente de casos esporádicos reportados en viajeros que regresan a Europa de regiones endémicas (31).

En 1987, A. cantonensis se identificó por primera vez en Norteamérica en roedores. Aunque el ensayo de PCR no detectó la lombriz intestinal como A. cantonensis en las muestras de los huéspedes intermediarios (caracoles) en Houston, Texas, el parásito ha sido identificado repetidas veces en los 
estados del sureste y del golfo de México, y, paralelamente, las infecciones humanas han aumentado. En 1995, se presentó un caso de una meningitis por $A$. cantonensis en un niño de 10 años de Nueva Orleans, Louisiana, que había comido caracoles crudos. Además, se informaron dos casos adicionales en niños de Houston en la primavera del $2016(31,32)$.

Al terminar el 2009, se reportaron casos y brotes de angiostrongiliasis en al menos nueve provincias de la República Popular China (457 casos detectados). Allí, las personas se infectan con $A$. cantonensis al consumir intencionalmente babosas y caracoles crudos o poco cocinados. La mayoría de los casos notificados provienen del sur del país y de otras partes del sudeste de Asia, especialmente el nordeste de Tailandia, donde los caracoles crudos o ligeramente cocidos se consideran un manjar (33).

Se ha demostrado que los viajeros que visitan áreas endémicas, particularmente aquellos que acampan, comen la cocina local, consumen moluscos crudos de agua dulce, o frutas y vegetales crudos, están en riesgo de contraer la infección por nematodos (34).

Desde 1945 se han reportado casi 3.000 casos en todo el mundo, la mayoría en el sur de China, pero el parásito se está extendiendo y ahora hay muchos más. En los Estados Unidos, casi todos los casos se han registrado en Hawái, donde se reportaron 104 casos entre 1959 y 2016. El parásito también está presente en los estados del sudeste. Además, a medida que el clima se calienta, es probable que este parásito tropical y subtropical se extienda aún más (35).

En Brasil se han reportado siete casos en humanos desde el 2007 en las regiones del sureste y el nordeste (36). En el 2014 se publicó una serie de 34 casos diagnosticados mediante ELISA y confirmados por RT-PCR (37). EI aumento de caracoles infectados se describió como una de las principales causas de la aparición de casos de meningoencefalitis eosinofílica en humanos (10).

Angiostrongylus cantonensis se ha reportado en 16 sitios geográficos a lo largo del continente americano, en seis especies huéspedes definitivas y en cinco especies accidentales. Del total de registros, solo en cuatro, tres en Brasil y uno en Haití, no se evidenció una coincidencia entre el hallazgo del huésped definitivo y el registro de la enfermedad. La mayoría de los casos de la enfermedad coinciden con el hallazgo definitivo del huésped. Sin embargo, en algunos lugares hay vacíos de información entre el informe del huésped definitivo y el registro de la enfermedad. La expansión de la población y los cambios climáticos invitan a ampliar y completar el rango de observación de las especies que implican posibles riesgos epidemiológicos (36).

En muchas áreas, principalmente tropicales, las poblaciones humanas han invadido ambientes naturales y sus condiciones socioeconómicas no permiten una atención médica adecuada, por lo cual muchos casos pueden haber pasado desapercibidos o no fueron reconocidos a lo largo de los años $(38,39)$.

La frecuencia de las infecciones por $A$. cantonensis en humanos probablemente no se notifica debido a una combinación de factores, incluido el frecuente curso autolimitado de la infección, la falta de conocimiento del parásito, la disponibilidad limitada de pruebas de diagnóstico y la falta de vigilancia nacional. Aunque en la mayoría de los casos la enfermedad se resuelve espontáneamente, las tasas de letalidad pueden alcanzar el $5 \%(40,41)$. 
La angiostrongiliasis cerebral debido a $A$. cantonensis sigue afectando la salud humana y la productividad en Tailandia. Los hábitos alimenticios de la población han sido un importante factor, particularmente en el nordeste del país, donde la enfermedad es endémica y los indígenas disfrutan de un plato local de caracoles poco cocidos llamado 'koi-hoi', generalmente como un aperitivo cuando ingieren bebidas alcohólicas después de sus actividades agrícolas diarias (42).

En la isla de Hainan en China, de las 118 ratas atrapadas durante las encuestas de campo, 13 ejemplares de $R$. norvegicus se encontraron infectadas con $A$. cantonensis. La tasa de infección fue de 11,02\%. Se examinaron 1.612 moluscos, $21,3 \%$ de los cuales albergaba larvas $L 3$ de $A$. cantonensis. Se recolectaron y analizaron 459 muestras de suero y 92 de ellas fueron positivas para $A$. cantonensis. La tasa global de anticuerpos positivos fue de $20,04 \%$ (43). En otro estudio en esta misma provincia, se detectó inmunoglobulina $\mathrm{G}(\mathrm{lgG})$ anti $A$. cantonensis en 20 de 393 (6\%) participantes, 12 de los cuales consumían habitualmente caracoles crudos (44).

En la provincia de Guangdong, 42 de 300 personas (14 \%) tenían anticuerpos IgG contra $A$. cantonensis, y cinco de ellas habían estado expuestas recientemente, según se comprobó al detectar lgM circulante (45).

En cuanto a la infección en humanos en Ecuador, hasta el 2009 se habían informado siete brotes en varias provincias del país, los cuales afectaron a 19 adultos y siete niños. Se estima que hasta hoy ha habido más de 87 casos humanos y tres muertes. En un artículo publicado por Guerrero, et al., en el 2008, se informó sobre la presencia del parásito en el tejido cerebral de uno de los pacientes fallecidos (46).

En 15 provincias de Ecuador se han encontrado caracoles infectados, con porcentajes de infección en $A$. fulica y Pomacea spp. de hasta $30 \%$ (datos del INSPI no publicados), además de un 36,5\% de ratas infectadas según estudios efectuados por el Centro de Referencia Nacional de Parasitología del INSPI en diferentes provincias.

En el primer estudio nacional sobre $A$. cantonensis en China, se estableció una serie de factores implicados en la aparición de casos humanos, a saber:

1. el área endémica de $A$. cantonensis es muy amplia y cubre siete provincias del sur, las cuales deben considerarse potencialmente endémicas;

2. la presencia de varios caracoles de agua dulce y terrestres en los mercados y restaurantes locales, pues se registraron caracoles $P$. canaliculata y $A$. fulica infectados con $A$. cantonensis que claramente estaban destinados al consumo humano, lo cual sugiere que la transmisión del parásito a los seres humanos se sigue produciendo y que las campañas de educación sanitaria y sensibilización iniciadas después del brote del 2006 en Pekín, dirigidas a los consumidores, el personal de salud y los funcionarios, deben mejorarse para detener el consumo de caracoles infectados;

3. Ios hábitos alimenticios de ciertos grupos étnicos aumentan el riesgo de infección por $A$. cantonensis; por ejemplo, el consumo de caracoles de agua dulce crudos o poco cocidos fue responsable del brote de angiostrongiliasis a principios del 2008 en Dali; por ello, los 
viajeros deberían estar mejor informados sobre los riesgos asociados con ciertos alimentos, y las regulaciones sobre su consumo e higiene deben aplicarse, y

4. las dos especies de moluscos invasores que facilitan la propagación y la transmisión de $A$. cantonensis en China son $P$. canaliculata y $A$. fulica, responsables de la endemia de este parásito (47).

La principal conclusión de este estudio fue que se amplió el número de provincias ecuatorianas estudiadas y se constató la presencia de $A$. cantonensis en ellas, lo cual confirma el carácter endémico de esta zoonosis en regiones urbanas y rurales del país. Rattus rattus y $R$. norvegicus son los huéspedes definitivos del parásito, lo que unido a la existencia de huéspedes intermediarios en casi todo el país, evidencia el potencial zoonótico de esta infección parasitaria. Es necesario muestrear las provincias restantes para establecer el alcance de la diseminación del parásito a lo largo del tiempo.

Estos estudios son esenciales para la implementación de las estrategias de vigilancia y control que reduzcan el riesgo de angiostrongiliasis entre los residentes locales y limiten la aparición de nuevos focos (47).

En los brotes reportados al Ministerio de Salud se ha involucrado el consumo de caracoles $A$. fulica y Pomacea spp. crudos, y si bien en su momento se lanzaron campañas locales destinadas a evitar dicho consumo, hace varios años que no se hacen actividades de promoción de salud.

En Ecuador, el consumo de caracoles crudos o poco cocidos no es habitual; se consumen caracoles ('churos') en ciertas celebraciones indígenas, pero en estudios hechos en dichos caracoles por el CRNP del INSPI (datos no publicados) no se detectó infección, presumiblemente por la altura en que se encuentran.

A diferencia de lo que sucede en los países asiáticos, como China y Tailandia, el consumo de caracoles no constituye una comida étnica o típica de ninguna de las provincias donde se han encontrado huéspedes intermediarios y definitivos infectados y casos de infección en humanos.

Esta parasitosis transmitida por alimentos presenta una gran incidencia en los países asiáticos debido a factores culturales (consumo de moluscos) que no están presentes en nuestro país, lo que explicaría que, a pesar de que en los huéspedes definitivos e intermediarios se han registrado tasas altas de infección, los casos humanos se presentan esporádicamente.

No se cuenta con una prueba serológica que permita detectar la presencia de anticuerpos, por lo que el diagnóstico sigue siendo clínico y epidemiológico. También hay que tener presente la variedad de presentaciones clínicas de la infección, que van desde un ligero malestar a una meningitis eosinofílica, por lo que la presencia de casos no diagnosticados debe siempre considerarse.

\section{Referencias}

1. Eamsobhana P. Eosinophilic meningitis caused by Angiostrongylus cantonensis - a neglected disease with escalating importance. Trop Biomed. 2014;31:569-78.

2. Martini RL. Diseño de un programa de control y prevención de Angiostrongylus cantonensis en el Ecuador período 2008-2009. Guayaquil: Universidad Estatal de Guayaquil; 2011. p. 1-19.

3. Chen TH. Un nouveau nemátode pulmonairé: Pulmonema cantonensis n. g. n. sp., de rats de Cantón. Ann Parasitol Hum Comp. 1935;13:321-7. 
4. Aguiar PH, Morera P, Pascual J. First record of Angiostrongylus cantonensis in Cuba. Am Trop Med Hyg. 1981;30:963-5. https://doi.org/10.4269/ajtmh.1981.30.963

5. Kliks M, Palumbo N. Eosinophilic meningitis beyond the Pacific Basin: the global dispersal of a peridomestic zoonosis caused by Angiostrongylus cantonensis, the nematode lungworm of rats. Soc Sci Med. 1992;34:199-212.

6. Kwon E, Ferguson T, Park S, Manuzak A, Qvarnstrom Y, Morgan S, et al. A severe case of Angiostrongylus eosinophilic meningitis with encephalitis and neurologic sequelae in Hawai'i. Hawaii J Med Public Health. 2013;72(Suppl.2):41-5.

7. Beaver PC, Jung R, Cupp EW. Beaver's Clinical Parasitology. Ninth edition. Philadelphia: Lea and Febiger; 1984. p. 292-4.

8. Del Risco U, Dieguez L. Presencia y distribución de hospederos intermediarios de Angiostrongylus cantonensis en Camagüey. Prevalencia e importancia epidemiológica para su control. AMC. 2004; 8(1):1-13.

9. Thiengo S, Fernandez M, Torres E, Coelho P, Lanfredi R. First record of a nematode Metastrongyloidea (Aelurostrongylus abstrusus larvae) in Achatina (Lissachatina) fulica (Mollusca, Achatinidae) in Brazil. J Invert Pathol. 2008;98:34-9. https://doi.org/10.1016/j.jip.2007.10.010

10. Maldonado A, Simões RO, Oliveira A, Motta E, Fernandez M, Pereira Z, et al. Firts report of Angiostrongylus cantonensis (Nematoda: Metastrongyloidae) in Achatina fulica (Mollusca: Gastropoda) from Southeast and South Brazil. Mem Inst Osw Cruz. 2010;105: 938-41. https://doi.org/10.1590/S0074-02762010000700019

11. Solórzano LF, Martini L, Muzzio J, Hernández H, Sarracent J, Rojas L. Angiostrongylus cantonensis: un parásito emergente en Ecuador. Rev Cub Med Trop. 2014;66:20-33.

12. Martini RL, Dorta CA. Angiostrongylus cantonensis - Emergencia en América. Primera edición. La Habana: Editorial Academia La Habana; 2016. p. 22-9.

13. Dard C, Piloquet JE, Qvarnstrom Y, Fox LM, M'kada H, Hebert JC, et al. First evidence of Angiostrongyliasis caused by Angiostrongylus cantonensis in Guadeloupe, Lesser Antilles. Am J Trop Med Hyg. 2017;96:692-7. https://doi.org/10.4269/ajtmh.16-0792

14. Barrientos Z. Aspectos básicos sobre la clasificación, recolección, toma de datos y conservación de los moluscos. Revista de Biología Tropical. 2003;51:13-30. https://doi.org/10.15517/rbt.v51i3.26363

15. Muzzio AJ. Moluscos hospederos intermediarios de Angiostrongylus cantonensis en dos provincias de Ecuador. La Habana: Instituto de Medicina Tropical "Pedro Kourí”; 2011. p. 28.

16. Wallace GD, Rossen L. Techniques for recovering and identifying larvae of Angiostrongylus cantonensis from molluscs. Hawaii: University of Hawaii at Manoa; 1969. p. 427-36.

17. Cowie RH. Biology, systematics, life cycle, and distribution of Angiostrongylus cantonensis, the cause of rat lungworm disease. Hawaii J Med Public Health. 2013;72:6-9.

18. Thiengo S, Maldonado A, Mota E, Torres E, Caldeira R, Carvalho OS, et al. The giant African snail Achatina fulica as natural intermediate host of Angiostrongylus cantonensis in Pernambuco, northeast Brazil. Acta Trop. 2010;115:194-9. https://doi.org/10.1016/j.actatropica.2010.01.005

19. Ibáñez CA, Berovides V, Hernández N, Cantillo J, De la Fuente J. Relación entre la morfofisiología y la carga de ectoparásitos en dos poblaciones de ratas (Rattus sp.) de La Habana. Rev Cubana Med Trop. 2012;64:224-34.

20. Ministerio de Salud, Instituto Nacional de Salud de Perú. Guía de manejo y cuidado de animales de laboratorio: ratón. Lima: Instituto Nacional de Salud; 2008. p. 5-9.

21. Stockdale WH, Slapcinsky J, Roff S, Mendieta CJ, Diaz GZ, Stern J, et al. Geographic distribution of Angiostrongylus cantonensis in wild rats (Rattus rattus) and terrestrial snails in Florida, USA. PLoS One. 2017;12:e0177910. https://doi.org/10.1371/journal.pone.0177910

22. Guerino LR, Pecora IL, Miranda MS, Aguiar-Silva C, Carvalho OS, Caldeira RL, et al. Prevalence and distribution of Angiostrongylus cantonensis (Nematoda, Angiostrongylidae) in Achatina fulica (Mollusca, Gastropoda) in Baixada Santista, São Paulo, Brazil. Rev Soc Bras Med Trop. 2017;50:92-8. https://doi.org/10.1590/0037-8682-0316-2016

23. Martin-Alonso A, Abreu-Yanes E, Feliu C, Mas-Coma S, Bargues MD, Valladares B, et al. Intermediate hosts of Angiostrongylus cantonensis in Tenerife, Spain. PLoS One. 2015;10:67. https://doi.org/10.1371/journal.pone.0120686 
24. Apichat V, Raxsina P, Seangchai N, Muncharee T. Survey of Angiostrongylus cantonensis in rats and giant African land snails in Phitsanulok province, Thailand. Asian Pac J Trop Med. 2011;4:597-9. https://doi.org/10.1016/S1995-7645(11)60154-5

25. Simões RO, Maldonado A, Olifiers N, García SJ, Bertolino FA, Luque LJ. A longitudinal study of Angiostrongylus cantonensis in an urban population of Rattus norvegicus in Brazil: The influences of seasonality and host features on the pattern of infection. Parasit Vectors. 2014;7:100. https://doi.org/10.1186/1756-3305-7-100

26. Wang Q, Lai D, Zhu X, Chen X, Lun Z. Human angiostrongyliasis. Lancet Infect Dis. 2008;8:621-30. https://doi.org/10.1016/S1473-3099(08)70229-9

27. Jarvi SI, Quarta S, Jacquier S, Howe K, Bicakci D, Dasalla C, et al. High prevalence of Angiostrongylus cantonensis (rat lungworm) on eastern Hawaii Island: A closer look at life cycle traits and patterns of infection in wild rats (Rattus spp.). PLoS One. 2017;12:e0189458. https://doi.org/10.1371/journal.pone.0189458

28. Monte TC, Simões RO, Oliveira AP, Novaes CF, Thiengo SC, Silva AJ, et al. Phylogenetic relationship of the Brazilian isolates of the rat lungworm Angiostrongylus cantonensis (nematoda: metastrongylidae) employing mitochondrial $\mathrm{COI}$ gene sequence data. Parasit Vectors. 2012;6:248-56. https://doi.org/10.1186/1756-3305-5-248

29. Carvalho Odos S, Scholte RG, Mendonça CL, Passos LK, Caldeira RL. Angiostrongylus cantonensis (nematode: metastrongyloidea) in molluscs from harbor areas in Brazil. Mem Inst Oswaldo Cruz. 2012;107:740-6. https://doi.org/10.1590/S0074-02762012000600006

30. Tokiwa T, Harunari T, Tanikawa T, Komatsu N, Koizumi N, Tung KC, et al. Phylogenetic relationships of rat lungworm, Angiostrongylus cantonensis, isolated from different geographical regions revealed widespread multiple lineages. Parasitol Int. 2012;61:431-6. https://doi.org/10.1016/j.parint.2012.02.005

31. Wang QP, Wu ZD, Wei J, Owen RL, Lun ZR. Human A. cantonensis: An update. Eur J Clin Microbiol Infect Dis. 2012;31:389-95. https://doi.org/10.1007/s10096-011-1328-5

32. Hammoud A, Nayes R, Murphy LS, Heresi RJ, Butler PG, Pérez JI, et al. Angiostrongylus cantonensis meningitis and myelitis, Texas, USA. Emerg Infect Dis. 2017;23:1037-8. https://doi.org/10.3201/eid2306.161683

33. Eamsobhana P, Yoolek A, Punthuprapasa P, Yong H. Effect of Thai 'koi-hoi' food flavoring on the viability and infectivity of the third-stage larvae of Angiostrongylus cantonensis (Nematoda: Angiostrongylidae). Act Trop. 2009;113:245-7. https://doi.org/10.1089/fpd.2008.0191

34. Ewers EC, Anisowicz SK. The potential danger of eating wild lettuce: A brief review of human rat lungworm infection. Hawaii J Med Public Health. 2014;73:28-32.

35. Cowie $\mathrm{RH}$. Angiostrongylus cantonensis: agent of a sometimes fatal globally emerging infectious disease (rat lungworm disease). ACS Chem Neurosci. 2017;8:2102-4. https://doi.org/10.1021/acschemneuro.7b00335

36. Thiengo SC, Simões OR, Fernandez MA., Maldonado A. Angiostrongylus cantonensis and rat lungworm disease in Brazil. Hawaii J Med Public Health. 2013;72:18-22.

37. Morassuti AL, Thiengo SC, Fernandez M, Sawanyawisuth K, Graeff-Teixeira C. Eosinophilic meningitis caused by Angiostrongylus cantonensis: an emergent disease in Brazil. Mem Inst Oswaldo Cruz. 2014;109:399-407. https://doi.org/10.1590/0074-0276140023

38. Valente R, Robles M, Navone TG, Diaz IJ. Angiostrongylus spp. in the Americas: Geographical and chronological distribution of definitive hosts versus disease reports. Mem Inst Oswaldo Cruz. 2018;113:143-52. https://doi.org/10.1590/0074-02760170226

39. Spratt DM. Species of Angiostrongylus (Nematoda: Metastrongyloidea) in wildlife: A review. Int J Parasitol Parasites Wildl. 2015;4:178-89. https://doi.org/10.1016/j.ijppaw.2015.02.006

40. Tseng YT, Tsai HC, Sy CL, Lee SS, Wann SR, Wang YH, et al. Clinical manifestations of eosinophilic meningitis caused by Angiostrongylus cantonensis: 18 years' experience in a medical center in southern Taiwan. J Microbiol Immunol Infect. 2011;44:382-9. https://doi.org/10.1016/j.jmii.2011.01.034

41. Defo AL, Lachaume N, Cuadro-Alvarez, E, Maniassom C, Martin E, Njuieyon, et al. Angiostrongylus cantonensis infection of central nervous system, Guiana Shield. Emerg Infect Dis. 2018;24:1153-5. https://doi.org/10.3201/eid2406.180168

42. Eamsobhana P. Angiostrongyliasis in Thailand: Epidemiology and laboratory Investigations. Hawaii J Med Public Health. 2013;72:28-32. 
43. Hu X, Du J, Tong C, Wang S, Liu J, Li Y, et al. Epidemic status of Angiostrongylus cantonensis in Hainan island, China. Asian Pac J Trop Med. 2011;4:275-7. https://doi.org/10.1016/S1995-7645(11)60085-0

44. Li YC, Hu XM, Tong CJ, Liu J, Li MT, Wang SQ. Investigation on serology, risk factor and awareness of Angiostrongylus cantonensis in Hainan province. Chinese journal of parasitology \& parasitic diseases. 2011;29:74-5.

45. Zhang Y, Huang D, Tan QM, Chen DX, Zhan XM. Epidemiological investigation of Angiostrongylus cantonensis in Jiangmen of Guangdong Province. Chinese journal of parasitology \& parasitic diseases. 2008;26:370-3.

46. Pincay T, García L, Narváez E, Decker O, Martini L, Moreira J. Angiostrongylosis due to Parastrongylus cantonensis in Ecuador. First report in South America. Trop Med Int Health. 2009;14 37.

47. Lv S, Zhang Y, Liu HX, Hu L, Yang K, Steinmann P, et al. Invasive snails and an emerging infectious disease: Results from the First National Survey on Angiostrongylus cantonensis in China. PLoS Negl Trop Dis. 2009;3:4-6. https://doi.org/10.1371/journal.pntd.0000368

48. Himsworth CG, Parsons KL, Jardine C, Patrick DM. Rats, Cities, people, and pathogens:A systematic review and narrative synthesis of literature regarding the ecology of ratassociated zoonoses in urban centers. Vector Borne Zoonotic Dis. 2013;13:1-11. https://doi.org/10.1089/vbz.2012.1195 